\title{
Consumo de alcohol y tabaco en estudiantes de enfermería ${ }^{1}$
}

\author{
Juan Yovani Telumbre-Terrero ${ }^{2}$ \\ Santiaga Enriqueta Esparza-Almanza ${ }^{3}$ \\ Bertha Alicia Alonso-Castillo ${ }^{4}$ \\ María Teresa de Jesús Alonso-Castillo ${ }^{5}$
}

Institución: Universidad Autónoma del Carmen

Universidad Autónoma de Nuevo León

\section{COMO CITAR}

\begin{abstract}
Telumbre-Terrero, J.Y., Esparza-Almanza, S.E., Alonso-Castillo, B.A y Alonso-Castillo, M.T.J (2016). Consumo de alcohol y tabaco en estudiantes de enfermería. Rev. Enfermería Actual en Costa Rica, 30, 1-16. DOI: http://dx.doi.org/10.15517/revenf.v0i30.22020
\end{abstract}

\section{RESUMEN}

Introducción. Los estudiantes de enfermería están inmersos en un contexto cultural tanto protector como de riesgo en cuanto al consumo de drogas, durante su proceso académico y/ o laboral, dado que enfrentan diversas situaciones físicas, emocionales y sociales que pueden aumentar la probabilidad de consumo con el fin de mitigar tales situaciones. El objetivo de la presente investigación fue determinar el tipo de consumo de alcohol y tabaco en estudiantes de enfermería.

Método. La población estuvo formada por 172 estudiantes de un programa de Licenciatura en Enfermería de dos universidades privadas del área Metropolitana de Monterrey Nuevo León, México. El diseño del estudio es no experimental de tipo transversal descriptivo. Se utilizó una cédula de datos personales y de consumo de alcohol y tabaco y el Cuestionario de Identificación de Desórdenes por Uso de Alcohol (AUDIT).

Resultados. Se identificó que predomina un tipo de consumo sensato de alcohol, seguido del dependiente y de uno dañino. En relación con el consumo de tabaco, prevalecieron los no fumadores, seguido de los fumadores experimentales, los exfumadores, los usuarios y los dependientes.

Conclusión. Estos resultados permiten comprender mejor la magnitud del problema del consumo de alcohol y tabaco entre los estudiantes universitarios, específicamente entre los estudiantes de enfermería quienes serán futuros profesionales de la salud y que actuarán como educadores y promotores para la adopción de estilos de vida saludables en la población general.

Palabras clave. Consumo-de-alcohol, consumo-de-tabaco, estudiante-de-enfermería.

\footnotetext{
${ }^{1}$ Fecha de recepción: 01 de julio del 2015 Fecha de aceptación: 15 de diciembre del 2015

${ }^{2}$ Maestro en Ciencias de Enfermería. Profesor y Coordinador de Servicio Social de la Licenciatura en Enfermería de la Facultad de Ciencias de la Salud de la Universidad Autónoma del Carmen, México. Correo Electrónico: jtelumbre@pampano.unacar.mx

${ }^{3}$ Maestra en Ciencias de Enfermería. Profesor y Sub directora de Pregrado de la Facultad de Enfermería de la Universidad Autónoma de Nuevo León (UANL), México. Correo electrónico: sesparza54@yahoo.com.mx

${ }^{4}$ Doctora en Bioética. Profesora de la Facultad de Enfermería de la Universidad Autónoma de Nuevo León, México. Correo electrónico: balonso mx@yahoo.com.mx.

${ }_{5}^{5}$ Doctora en Bioética. Profesora y colaboradora del Cuerpo Académico de Prevención de Adicciones de la Facultad de Enfermería de la Universidad Autónoma de Nuevo León, México. Correo electrónico: talonso 55@,hotmail.com
} 


\title{
Consumption of alcohol and tobacco in students of nursing ${ }^{1}$
}

Institution: University Autónoma del Carmen

University Autónoma de Nuevo León

\section{CITED:}

\author{
Juan Yovani Telumbre-Terrero ${ }^{2}$ \\ Santiaga Enriqueta Esparza-Almanza ${ }^{3}$ \\ Bertha Alicia Alonso-Castillo ${ }^{4}$ \\ María Teresa de Jesús Alonso-Castillo ${ }^{5}$
}

Telumbre-Terrero, J.Y., Esparza-Almanza, S.E., Alonso-Castillo, B.A y Alonso-Castillo, M.T.J (2016). Consumption of alcohol and tobacco in students of nursing. Rev. Enfermería Actual de Costa Rica, 30, 1-16. DOI: http://dx.doi.org/10.15517/revenf.v0i30.22020

\section{SUMMARY}

Introduction. Nursing students are immersed in a risky and protective cultural context on drugs consumption, during their academic term and/or work as they face various physical, emotional and social situations that may increase the likelihood of consuming alcohol and tobacco as a way to mitigate these situations. The objective of this research was to determine the type of alcohol and tobacco consumption among nursing students.

Method. The population consisted of 172 students of a Nursing program of two private universities in the metropolitan area of Monterrey, Nuevo Leon, Mexico. The design of this study is non-experimental crosssectional. A Personal Data Document, which also included Alcohol and Tobacco consumption and a Questionnaire to identify Disorders for Alcohol consumption (AUDIT), was used.

Results. It was identified that prudent consumption is predominant, followed by the dependent and harmful consumption. As for tobacco consumption, nonsmokers were predominant, followed by the experimental smokers, former smokers, users and dependent smokers.

Conclusion. These results allow a better understanding about the magnitude of alcohol and tobacco consumption problem among college students, specifically among nursing students who will be future health professionals and act as educators and advocates for the adoption of healthy lifestyles in the general population.

Keywords. Alcohol-consumption, Nursing-student, Tobacco-consumption.

\footnotetext{
${ }^{1}$ Date of acceptance: July 01, 2015

Date of receipt: December 15, 2015

${ }^{2}$ Master of Science in Nursing. Professor and Coordinator of Social Service of Nursing, Faculty of Health Sciences of the University Autonoma del Carmen, Mexico. Email: jtelumbre@pampano.unacar.mx

${ }^{3}$ Master of Science in Nursing. Undergraduate Professor and Deputy Director of the School of Nursing of the Autonomous University of Nuevo Leon (UANL), México. Email: sesparza54@yahoo.com.mx

${ }^{4}$ Doctor in Bioethics. Professor of the School of Nursing of the Autonomous University of Nuevo Leon, México. Email: balonso mx@yahoo.com.mx.

${ }^{5}$ Doctor in Bioethics. Professor of the School of Nursing of the Autonomous University of Nuevo Leon, México. Email: talonso 55@hotmail.com
} 


\section{Revista Electrónica Enfermeria Actual en costa Rica}

\section{INTRODUCCIÓN}

Uno de los factores de riesgo para la salud a nivel global es el consumo de drogas: al respecto, según la Organización Mundial de la Salud (OMS, 2009) el consumo de alcohol y tabaco representa un factor significativo en la tasa de morbilidad general en el mundo, debido a las consecuencias biológicas, psicológicas y sociales que ocasiona en el individuo, las familias y los colectivos. La carga estimada atribuible es de 59 millones de Años de Vida Ajustado por Discapacidad (AVAD) por consumo de tabaco, 58 millones de AVAD por consumo de alcohol y 11 millones de AVAD por consumo de drogas ilícitas, es decir, el tabaco y el alcohol representan el 4,1\% y 4\%, respectivamente de la carga de morbilidad, en tanto que las drogas ilícitas representaron solo el 0,8\% (OMS, 2009; Organización Panamericana de la Salud [OPS], 2009).

En México, la Encuesta Nacional de Adicciones (ENA, 2011) señala que el consumo de alcohol alguna vez en la vida en población de 12 a 65 años fue de $42,9 \%$ mientras que, en el último año fue de $30 \%$ y en el último mes, de 14,5\%. El 14,5\% de la población presenta un consumo alto de alcohol: el $0,2 \%$ refirió un consumo diario de alcohol, el $1 \%$ está formado por consumidores consuetudinarios y un $4,1 \%$ se refiere a adolescentes con dependencia al alcohol (Instituto Nacional de Psiquiatría Ramón de la Fuente Muñiz, Instituto Nacional de Salud Pública [INSP] y Secretaría de Salud [SSA], 2011).

Por su parte, la Encuesta Nacional de Salud y Nutrición (ENSANUT, 2012) reporta una prevalencia de consumo de tabaco de $9,2 \%$, representado por un $12,3 \%$ para los hombres y un $6 \%$ para las mujeres; en los jóvenes la prevalencia de consumo diario de tabaco es de $2,6 \%$, consumen en promedio 3.7 cigarros al día y un 6,6\% fuma su primer cigarro en los primeros 30 minutos después de levantarse (Instituto Nacional de Salud Pública [INSP] y SSA, 2012).

Tras exponer lo anterior, el presente estudio centra su atención en estudiantes de enfermería quienes además de encontrarse en la etapa de la juventud, son futuros profesionales de la salud y están inmersos en un contexto cultural tanto protector como de riesgo en cuanto al consumo de drogas (Pinos, Inocenti y Renato, 2008). $\mathrm{Al}$ respecto, se destaca que, aún y cuando no han concluido su proceso académico, el estudiante de enfermería, en ocasiones, ya se encuentra trabajando en el área de la salud y es probable que enfrente situaciones como desgaste físico y emocional, excesiva carga horaria, mayor responsabilidad laboral, privación de la convivencia familiar y diversión, factores que pueden aumentar la probabilidad de que consuma alcohol y tabaco como una forma de mitigar tales situaciones (Barbosa y Ferreira, 2008; Braga y Bastos, 2004).

En este sentido, la evidencia muestra que del $30 \%$ al $98 \%$ de estudiantes de enfermería ha consumido alcohol alguna vez en la vida, quienes practican un consumo de bajo riesgo en la mayoría $(29,3 \%$ al $39,5 \%)$ de, seguido de uno dañino (10,7\%-14\%) y uno dependiente en una menor proporción $(6,3 \%-9,1 \%)$, entre quienes se reporta diferencias en el consumo por sexo $\left(\chi^{2}=9,697 ; p=, 008\right)$, el cual encabezan los hombres, con un 58,6\%, seguido de las mujeres, con un $35,3 \%$ ). La frecuencia de consumo es de dos veces por semana, acompañado de la ingesta de hasta diez bebidas alcohólicas en una sola ocasión (López, Villar y Gherardi, 2011; Matute y Pillon, 2008; Tam y Benedita, 2010).

En relación con consumo de tabaco, se identificó que en los estudiantes de enfermería el consumo de tabaco es ocasional, y que puede variar por sexo, estado civil y grado escolar: es mayor en los hombres (hombres 


\section{Revista Electrónica Enfermeria Actual en costa Rica}

$53,1 \%$ y mujeres $37,2 \%)$, así como en aquellos estudiantes solteros $(43,2 \%)$ y que cursan un mayor grado escolar (44,4\%) (López y al., 2011; Montoya, Cunningham, Brans, Strike y Miotto, 2009; Rodríguez, Pineda y Vélez, 2010). Con base en lo anterior, este estudio persigue determinar el tipo de consumo de alcohol y tabaco en estudiantes de enfermería, con cuyos resultados se espera determinar los grupos prioritarios para participar en intervenciones de prevención primaria y secundaria del consumo de drogas licitas como alcohol y tabaco.

\section{MATERIAL Y MÉTODO}

La población estuvo formada por 172 estudiantes de un programa de Licenciatura en Enfermería de dos universidades privadas del área Metropolitana de Monterrey, Nuevo León. El tipo de muestreo se llevó a cabo mediante un censo, es decir, se contó con la participación de todos los estudiantes de ambas escuelas; sin embargo, se consideró 166 participantes debido a que seis de ellos no se encontraban al recolectar los datos. El diseño del estudio es no experimental de tipo transversal, porque se realizó una sola medición en el tiempo, y descriptivo (Burns y Grove, 2004), porque se describió el consumo de alcohol y tabaco de los estudiantes enfermería tal y como ocurre de manera natural. Para la recolección de los datos se utilizó una cédula de datos personales y de consumo de alcohol y tabaco, con el fin de conocer datos como la edad, sexo, estado civil, semestre, ocupación y tipo de ocupación (la cual fue elaborada por los investigadores, cuyo referente fueron las características de los participantes, además de que fue el primer cuestionario contestado por los participantes) y el Cuestionario de Identificación de Desórdenes por Uso de Alcohol (AUDIT).

Respecto del consumo de alcohol se utilizó el AUDIT, desarrollado por un grupo de expertos de la Organización Mundial de la Salud [OMS], y validado en población Mexicana por De la Fuente y Kersenobich (1992): consta de 10 reactivos que examinan el consumo de alcohol durante los últimos doce meses, así como sus consecuencias. El AUDIT consta de tres dominios: los reactivos 1, 2 y 3 determinan la cantidad y frecuencia del consumo; los reactivos del 4 al 6 evalúan la posibilidad de que exista dependencia al alcohol y los reactivos 7, 8, 9 y 10 exploran el consumo dañino de alcohol.

La puntuación mínima del cuestionario es de 0 y la máxima, de 40: las puntuaciones de 1 a 3 son consideradas un consumo sensato; de 4 a 7 , consumo dependiente y de 8 puntos a 40, dañino. Los autores de este instrumento reportan una sensibilidad de 80\% y una especificidad del 89\% (De la Fuente y Kersenobich, 1992). Este cuestionario ha sido utilizado específicamente en estudiantes de enfermería con un Alpha de Cronbach de $\alpha=$ 0.84 (López, 2012) que en este estudio fue de 0.77, lo cual se considera una consistencia interna aceptable (Burns y Grove, 2004; Polit y Hungler, 1999). Por otra parte, fue necesario indagar sobre el consumo de alcohol: alguna vez en la vida, en el último año, en el último mes, en los últimos siete días. Se preguntó además la edad de inicio de consumo de alcohol, así como la cantidad de bebidas alcohólicas en un día típico.

Se incluyó también el consumo tabaco alguna vez en la vida, en el último año, en el último mes, en los últimos siete días, la edad de inicio del consumo de tabaco y la cantidad de cigarros consumidos en un día típico. Para identificar el tipo de fumador se utilizó la clasificación propuesta por Tapia (2001), quien categoriza a los sujetos en exfumador, fumador experimental, usuario o dependiente tomando en cuenta la cantidad y la frecuencia del consumo de tabaco a través de una pregunta con cuatro opciones de respuesta: si el participante ha fumado en el pasado y actualmente no fuma se consideró ex fumador; si fumó menos de cien cigarros en su vida y consume ocasionalmente, es fumador experimentador; en caso de que haya fumado más de cien cigarros en su vida y fume 
a diario, es usuario, y si actualmente fuma a diario y el primer cigarro lo consume en un lapso menor de 30 minutos después de haberse despertado, es fumador dependiente (Tapia, 2001).

\section{Procedimiento de recolección de datos}

Para la recolección de datos primeramente se solicitó la evaluación del estudio por parte de las Comisiones de Ética e Investigación de la Facultad de Enfermería de la Universidad Autónoma de Nuevo León. Una vez obtenida su aprobación se solicitó autorización por escrito a los directivos de las Instituciones donde se llevó a cabo el estudio y se solicitó las listas de los grupos.

Una vez identificados los grupos, se contactó al área de prefectura para solicitar los horarios y aulas donde se localizaban los estudiantes. Posteriormente, se visitó a los grupos en el horario señalado por la institución, de tal manera que no interfirió con su carga académica y se le solicitó al maestro de clase su autorización para hablar con los estudiantes e invitarlos a participar en el estudio (se les explicó de forma clara y sencilla los objetivos de la investigación). A los alumnos mayores de edad que aceptaron participar se les entregó un consentimiento informado, el cual fue leído, respondido y firmado.

Al alumno menor de edad se le entregó un consentimiento informado para el padre o tutor y se acordó fecha, lugar y hora para recogerlo: a quien se le autorizó participar en el estudio se le explicó nuevamente el propósito del estudio y se le entregó el asentimiento informado para que lo leyera y firmara, de manera que pudiera participar en el estudio. A cada participante se le brindó un sobre con los instrumentos, los cuales fueron completados en aproximadamente 20 minutos. Al concluir el llenado de los instrumentos, se les solicitó a los participantes colocarlos dentro del sobre y depositarlos en un contenedor colocado frente del aula. Finalmente se agradeció la participación.

\section{Consideraciones éticas.}

El presente estudio se apegó a lo dispuesto en el Reglamento de la Ley General de Salud (LGS) en Materia de Investigación para la Salud en seres Humanos en México (SSA, 1987). Se consideró el Título Segundo de los aspectos éticos de la investigación en seres humanos, Capítulo I, Artículo 13, 14, 16, 17, 20, 21, 36,57 y 58.

\section{Análisis de datos}

Los datos fueron procesados en el programa estadístico Statistical Package for the Social Sciences (SPSS) versión 17.0 para Windows. La consistencia interna del instrumento fue determinada a través del Coeficiente de Alpha de Cronbach. Se utilizó la estadística descriptiva para obtener frecuencias, proporciones, medidas de tendencia central y de variabilidad. 


\section{RESULTADOS}

En relación con las características sociodemográficas de los participantes del estudio, se identificó que el $68,7 \%(n=114)$ de la muestra corresponde al sexo femenino. En cuanto a la edad, el $62 \%(n=103)$ de $10 \mathrm{~s}$ estudiantes tiene entre 17 y 21 años. Respecto del grado escolar, el 40,4\% $(n=67)$ cursa el primer año y el 36,7\% $(n=61)$ el segundo año. El 36,7\% $(n=61)$ de los estudiantes de enfermería estudia y trabaja y de ese porcentaje, el $96,7 \%(n=59)$ realiza actividades de tipo terciario; es decir, se desempeña en el comercio, restaurantes, alojamiento, transportes, eventos sociales.

Los participantes presentaron una media de edad de 21.4 años $(D E=4.3)$, en promedio iniciaron el consumo de alcohol a los 16.6 años $(D E=2.4)$, además de que se reporta que los participantes consumen en promedio $5.3(D E=4.6)$ bebidas alcohólicas en una sola ocasión. En lo concerniente al AUDIT, la media más alta fue para el consumo sensato $(\bar{x}=3.9, D E=2.5)$, seguida del consumo dañino $(\bar{x}=1.1, D E=2.0)$ y del dependiente $(\overline{\mathrm{x}}=.6, D E=.9$ ). Se identificó que la media de edad de inicio del consumo de tabaco fue a los 16.2 años $(D E=2.9)$ y los participantes consumen en promedio $3.9(D E=5.3)$ cigarros en una sola ocasión (tabla1).

Tabla 1. Monterrey, Nuevo León: Distribución de estudiantes de enfermería según datos sociodemográficos y de consumo de alcohol y tabaco, 2015.

\begin{tabular}{l|c|c|c|c|c|c}
\hline Variable & & & & & \multicolumn{3}{|c}{ Valor } \\
\cline { 5 - 7 } & $N$ & $\bar{x}$ & Mdn & DE & Mínimo & Máximo \\
\hline Edad en años & 166 & 21.4 & 20.0 & 4.3 & 17 & 46 \\
Edad de inicio del consumo de alcohol & 138 & 16.6 & 17.0 & 2.4 & 11 & 24 \\
Cantidad de bebidas consumidas * & 122 & 5.3 & 4.0 & 4.6 & 1.0 & 25 \\
AUDIT general ** & 122 & 5.7 & 5 & 4.6 & 0 & 21 \\
$\quad$ Consumo sensato** & 122 & 3.9 & 4 & 2.5 & 0 & 11 \\
$\quad$ Consumo dependiente** & 122 & .6 & .00 & .9 & 0 & 4 \\
$\quad$ Consumo dañino** & 122 & 1.1 & .00 & 2.0 & 0 & 10 \\
Edad de inicio del consumo de tabaco* & 93 & 16.2 & 16 & 2.9 & 11 & 30 \\
Cantidad de cigarros** & 69 & 3.9 & 3 & 5.3 & 1 & 40 \\
\hline
\end{tabular}

$n=$ muestra, $\overline{\mathrm{x}}=$ media, $\mathrm{Mdn}=$ mediana, $\mathrm{DE}=$ desviación estándar, AUDIT $=$ Cuestionario de identificación de trastornos por uso de alcohol, *personas que consumieron alcohol y tabaco alguna vez en la vida, **consumidores de alcohol y tabaco en el último año.

Fuente: elaboración propia

Se identificó que el $83,1 \%(n=138)$ de los estudiantes de enfermería ha consumido alcohol alguna vez en su vida: $73,5 \%(n=122)$ en el último año, $53 \%(n=88)$ en el último mes y el 33,7\% $(n=56)$ en los últimos siete días. Así también del total de los estudiantes de enfermería, el 56\% $(n=93)$ ha consumido tabaco alguna vez en la vida, un $41,6 \%(n=69)$ lo ha consumido en el último año, un 30,7\% $(n=51)$ en el último mes y el $23,5 \%(n=39)$ en los últimos siete días (tabla 2). 
Tabla 2. Monterrey, Nuevo León. Distribución de estudiantes de enfermería según prevalencia global, actual e instantánea de consumo de alcohol y tabaco, 2015.

\begin{tabular}{lcccccc}
\hline & \multicolumn{2}{c}{$\mathrm{Si}$} & \multicolumn{2}{c}{ No } & \multicolumn{2}{c}{ IC 95\% } \\
\cline { 2 - 7 } & $h i$ & $H i$ & $h i$ & $H i$ & $L I$ & $L S$ \\
\hline Consumo de alcohol & & & & & & \\
\hline Alguna vez en la vida & 138 & 83,1 & 28 & 16,9 & 77 & 89 \\
En el último año & 122 & 73,5 & 44 & 26,5 & 67 & 80 \\
En el último mes & 88 & 53,0 & 78 & 47,0 & 45 & 61 \\
En los últimos siete días & 56 & 33,7 & 110 & 66,3 & 26 & 41 \\
\hline Consumo de tabaco & & & & & & \\
\hline Alguna vez en la vida & 93 & 56,0 & 73 & 44,0 & 48 & 64 \\
En el último año & 69 & 41,6 & 97 & 58,4 & 34 & 49 \\
En el último mes & 51 & 30,7 & 115 & 69,3 & 24 & 38 \\
En los últimos siete días & 39 & 23,5 & 127 & 76,5 & 17 & 30 \\
\hline
\end{tabular}

$h i=$ Frecuencias absolutas, $H i=$ frecuencias relativas, $I C=$ intervalo de confianza para el $\%$ de respuestas afirmativas, $L I=$ límite inferior, $L S=$ límite superior, $N=166$.

Fuente: elaboración propia

En lo que respecta a los alumnos que han consumido alcohol en el último año, y tomando como referente los tipos de consumo de alcohol de acuerdo al AUDIT, se identificó que el 36,9\% $(n=45)$ de los estudiantes de enfermería practica un consumo sensato de alcohol; el 32,8\% $(n=40)$, un consumo dependiente, y el 30,3\% $(n=37)$, un consumo dañino (tabla 3).

Tabla 3. Monterrey, Nuevo León. Distribución de estudiantes de enfermería según consumo de alcohol (AUDIT), 2015.

\begin{tabular}{ccc}
\hline Tipo de consumo de alcohol & $H i$ & $H i$ \\
\hline Consumo sensato & 45 & 36,9 \\
Consumo dependiente & 40 & 32,8 \\
Consumo dañino & 37 & 30,3
\end{tabular}

$h i=$ Frecuencias absolutas, $H i=$ frecuencias relativas, AUDIT= Cuestionario de identificación de trastornos debidos al consumo de alcohol, $n=122$.

Fuente: elaboración propia 
La tabla 4 muestra los tipos de consumo de tabaco donde se observa que el $44 \%(n=73)$ de los estudiantes de enfermería nunca han consumido tabaco, $14,5 \%(n=24)$ son ex fumadores, $27,7 \%(n=46)$ experimentadores, $10,8 \%(n=73)$ usuarios y el $3 \%(n=5)$ presenta dependencia al tabaco.

Tabla 4. Monterrey, Nuevo León. Distribución de estudiantes de enfermería según consumo de tabaco, 2015.

\begin{tabular}{ccc}
\hline Tipo de fumador & $h i$ & $H i$ \\
\hline No fumador & 73 & 44,0 \\
Ex fumador & 24 & 14,5 \\
Experimentador & 46 & 27,7 \\
Usuario & 18 & 10,8 \\
Dependiente & 5 & 3,0 \\
\hline
\end{tabular}

$h i=$ Frecuencias absolutas, $H i=$ frecuencias relativas, $\mathrm{N}=166$.

Fuente: elaboración propia

\section{DISCUSIÓN}

Se identificó que los participantes empiezan el consumo de alcohol y tabaco en promedio a los 16 años de edad, lo cual concuerda con Rodríguez et al (2010) y con los datos de la Encuesta Nacional de Adicciones (2011), los cuales indican que el consumo de alcohol o tabaco se inicia a los 17 años o menos, lo cual podría deberse a que los jóvenes se encuentran en un periodo de transición entre la adolescencia y la adultez, en el que se sienten miembros y partícipes de una "cultura de edad" caracterizada por sus propios comportamientos, valores, normas, espacios y modas.

Los estudiantes de enfermería consumen en promedio cinco bebidas alcohólicas y tres cigarros por ocasión, tendencia similar a los resultados de López (2012) y Navarro, Espig y Medina (2010) y que es preocupante, puesto que son estudiantes de enfermería de quienes se espera que sean modelos en su desarrollo profesional para otros estudiantes y para los pacientes que atienden (Rodríguez, Pineda y Vélez, 2010).

El consumo de alcohol alguna vez en la vida y en último año fue menor en comparación con los hallazgos de Montoya (2009); sin embargo, el consumo de alcohol en el último mes y en los últimos siete días fue mayor en relación con los hallazgos de Bautista et al (2011), quienes reportan prevalencias inferiores al 15\% en estudiantes de enfermería de El Salvador, situación que puede vincularse con la interacción de variables sociodemográficas como el sexo, edad, nivel socioeconómico, nivel educativo, estado civil y lugar de residencia, así como por variables ambientales y culturales propias del contexto universitario que facilitan y promueven el consumo excesivo de alcohol (Díaz, Díaz, Hernández, Narro, Fernández y Solís, 2008).

El consumo de tabaco alguna vez en la vida es similar al reportado por Montoya (2009), quien identificó una prevalencia global de 59\% en estudiantes de enfermería de Medellín, Colombia; el consumo de tabaco en el último año, mes y siete días fue mayor en comparación con lo señalado por Bautista et al (2011) y Rodríguez, 


\section{Revista Electrónica Enfermeria Actual en costa Rica}

Pineda y Vélez (2010) quienes reportan prevalencias de consumo menores al 10\% en estudiantes de enfermería de El Salvador y en Antioquia, Colombia.

Los hallazgos del presente estudio son relevantes, máxime al estar formada la población por estudiantes de enfermería, quienes conocen lo que provoca el consumo de tabaco. En ese sentido, Díaz et al (2011), tras revisar la literatura en Brasil respecto del consumo de drogas y su vinculación con el profesional de enfermería, encontraron que los estudiantes de enfermería enfrentan diversas situaciones en el contexto académico y clínico que pueden provocar estrés, irritabilidad, ansiedad, cansancio y cambios en los patrones de sueño, los cuales son factores de riesgo que propician el consumo de tabaco y otras drogas como una forma de disminuir estados emocionales negativos, situación que también refleja la falta de mecanismos positivos de afrontamiento ante estas situaciones. En México, no se identificó literatura que permita establecer que el contexto de la población estudiantil de enfermería en Brasil sea similar o diferente.

Referente al tipo de consumo de alcohol, se identificó que los resultados difieren con López et al (2011) y Martínez et al (2010), quienes reportan que entre el $67 \%$ y $88 \%$ de los estudiantes presenta un consumo sensato de alcohol, seguido del consumo dependiente ( $10 \%$ y $8,7 \%$ respectivamente) y dañino ( $2 \%$ y $3,1 \%$ respectivamente) en menores proporciones en estudiantes de enfermería de Brasil y Colombia.

En el área de la salud, se constata que el estudiante o profesional de enfermería enfrenta diversas situaciones en la vida personal y profesional, posibilidad que también debe ser considerada para el estudiante del enfermería puesto que, a pesar de ser un trabajador temporal, está sujeto a desgaste psicofísico por actividades académicas y por convivir con riesgos de tipo ergonómico, psicosocial y organizacional en el ambiente laboral que se considera causas o efectos en cuanto al consumo de alcohol, tabaco y otras drogas (Costa, Gollner, Ribeiro, Spinola y Bertolossi, 2009), hasta provocar un consumo dependiente o dañino (Barbosa y Ferreira, 2008).

En cuanto al tipo de fumador, los hallazgos concuerdan con Rodríguez, Pineda y Vélez (2010) y con Sánchez y Pillon (2011) quienes consideran que hay que tener en cuenta que el paso por la universidad genera ansiedad en los estudiantes, debido a que los estudios y exámenes pueden ocasionar que se inicie, consolide o aumente el hábito de tabaco, por lo tanto el espacio universitario puede convertirse en un factor de riesgo o uno protector en el consumo de tabaco (Morales, Díaz, Garrido y Pascual, 2011).

\section{CONCLUSIÓN}

Los resultados aportan información veraz y oportuna en cuanto al perfil del consumo de alcohol y tabaco en estudiantes de enfermería de Monterrey Nuevo León, México.

Se evidenció que el tipo de consumo de alcohol es de tipo sensato, lo que indicaría que su formación puede ser un factor protector ante el consumo de esta sustancia. Además se concluye que el mayor grupo de estudiantes son no fumadores, situación que afirma lo mencionado anteriormente.

Estos resultados contribuyen a comprender mejor la magnitud del consumo de alcohol y tabaco entre los estudiantes universitarios, específicamente entre los profesionales de enfermería quienes serán futuros 


\section{Revista Electrónica Enfermeria Actual en costa Rica}

profesionales de la salud, así como educadores y motivadores para la adopción de estilos de vida saludables por la población.

\section{REFERENCIAS}

Barbosa, D y Ferreira, F. (2008). El trabajo del estudiante de enfermería como un factor de riesgo para el consumo de alcohol y otras drogas. Revista Latino-am Enfermagem, 16 (número especial).

Bautista, P., Simich, L., Strike, C., Brandns, B., Giesbrecht, N. y Khenti, A. (2011). Policonsumo simultáneo de drogas en estudiantes de pregrado del área de la salud de una Universidad de el Salvador. Texto \& Contexto Enfermegen, 21.

Braga, A. y Bastos A. (2004). Formação do acadêmico de enfermagem e seu contato com as drogas psicoativas. Revista Texto \& Contexto Enfermegen, 13 (2), 241-249.

Burns, N. y Grove, S.K. (2004). Investigación en Enfermería (3 ${ }^{\mathrm{a}}$ Ed.) España: Elsevier.

De la Fuente, J.R. y Kershenobich, D (1992). El alcoholismo como problema médico. Revista de la Facultad de Medicina, 35 (2), 45-51.

Díaz, L., Marina, C., Taubert, F., Amorim, L., Barcelos, C., Valenzuela, V. Y Cruz, M. (2011). Drug use in nursing workers. Ciencia y Enfermería. 17 (2), 37-45.

Díaz, M. A., Díaz, M., Hernández, C., Narro, R., Fernández, V. y Solís, T. C. (2008). Prevalencia del consumo riesgoso y dañino de alcohol y factores de riesgo en estudiantes universitarios de primer ingreso. Salud mental, 31(4), 271-282.

Instituto Nacional de Psiquiatría Ramón de la Fuente Muñiz; Instituto Nacional de Salud Pública [INSP]; Secretaría de Salud [SSA]. (2011). Encuesta Nacional de Adicciones [ENA]: Reporte de Alcohol.

Instituto Nacional de Salud Pública [INSP] y Secretaría de Salud [SSA] (2012). Encuesta Nacional de Salud y Nutrición [ENSANUT]. Resultados Nacionales.

López, M. (2012). Creencias y actitudes de los estudiantes de enfermería ante el paciente que consume alcohol y el consumo de alcohol. (Tesis de Maestría inédita). Facultad de Enfermería. Universidad Autónoma de Nuevo León México.

López, M., Villar, L. y Gherardi, E. (2011). Consumo de drogas lícitas en estudiantes de enfermería de Bogotá, Colombia. Revista Latino-am Enfermagem, 19 (número especial), 707-713.

Martínez, R., Coronado, H., Betancourt, E., Díaz, O. y Gallegos, M. (2010). Drogas licitas e ilícitas: consumo de los estudiantes de la Facultad de Enfermería. Revista de Enfermería Neurológica, 10 (3), 130-134. 
Costa, E.R, Gollner, R.C., Ribeiro, M.T.., Spinola, T. y Bertolossi C. (2009). Concepções do trabalhador de enfermagem sobre drogas: a visibilidade dos riscos. R Enferm UERJ. 17 (3): 368-372.

Matute, R. y Pillon, S. (2008). Uso de bebidas alcohólicas entre estudiantes de enfermería en Honduras. Revista Latino-am Enfermagem, 16 (número especial).

Montoya, V., Cunningham, J., Brans, B., Strike, C. y Miotto, W. (2009). Consumo percibido y uso de drogas lícitas e ilícitas en estudiantes universitarios de Medellín, Colombia. Revista Latino-am Enfermagem, 17, 886-892.

Morales, D., Díaz, B., Garrido, D.. y Pascual, O. (2011). Autoeficacia y consumo de tabaco en estudiantes universitarios. Apuntes de Psicología. 29 (3), 459-470.

Navarro, D., Espig, H. y Medina, V. (2010). Tobacco consumption in university students entering health sciences schools. Revista de Salud Pública. 14 (1), 54-61.

Organización Panamericana de la Salud [OPS] y Organización Mundial de la Salud [OMS]. (2009). Epidemiología del uso de drogas en América Latina y el Caribe: un enfoque de Salud Pública. 1-36.

Pinos, P., Inocenti, M., Renato, T. (2008). Consumo de Benzodiacepinas sin prescripción médica en los/as estudiantes de primer año de la escuela de enfermería de la Universidad de Guayaquil, Ecuador. Revista Latino-am Enfermagem, 16 (número especial).

Polit, D. y Hungler, B. (1999). Investigación científica en ciencias de la salud (6ª . ed.). México: Mc Graw-Hill Interamericana.

Rodríguez, G., Pineda, B. y Vélez, Y. (2010). Características del consumo de tabaco en estudiantes de enfermería de la Universidad de Antioquia, Colombia. Revista Investigación y Educación en Enfermería. 28 (3), 370383.

Sánchez, C. y Pillon, S. (2011). Tabaquismo entre universitarios: caracterización del uso en la visión de los estudiantes. Rev. Latino-Am. Enfermagem. 19 (Número especial), 730-737.

Secretaría de Salud [SSA] (1987). Reglamento de la Ley general de salud en materia de investigación para la salud.

Tam, E. y Benedita, C. (2010). El consumo de alcohol y el estrés entre estudiantes del segundo año de enfermería. Revista Latino-am Enfermagem, 18 (número especial), 496-503.

Tapia, C. (2001). Las adicciones: dimensiones, impacto y perspectiva. (2da Ed.). México, D.F.: Manual Moderno. 